\title{
A Novel Feature Selection Approach Based on FODPSO and SVM
}

\author{
Pedram Ghamisi, Student Member, IEEE, Micael S. Couceiro, Member, IEEE, \\ Jon Atli Benediktsson, Fellow, IEEE
}

\begin{abstract}
A novel feature selection approach is proposed to address the curse of dimensionality and reduce the redundancy of hyperspectral data. The proposed approach is based on a new binary optimization method inspired by the FractionalOrder Darwinian Particle Swarm Optimization (FODPSO). The overall accuracy of a Support Vector Machine (SVM) classifier on validation samples is used as fitness values in order to evaluate the informativity of different groups of bands. In order to show the capability of the proposed method, two different applications are considered. In the first application, the proposed feature selection approach is directly carried out on the input hyperspectral data. The most informative bands selected from this step are classified by SVM. In the second application, the main shortcoming of using attribute profiles for spectral-spatial classification is addressed. In this case, a stacked vector of the input data and an attribute profile with all widely used attributes is created. Then, the proposed feature selection approach automatically chooses the most informative features from the stacked vector. Experimental results successfully confirm that the proposed feature selection technique works better in terms of classification accuracies and CPU processing time than other studied methods without requiring the number of desired features to be set $a$ priori by users.
\end{abstract}

Index Terms-Hyperspectral Image Analysis, Spectral-Spatial Classification, Attribute Profile, Feature Extraction, Random Forest Classifier, Automatic Classification.

\section{INTRODUCTION}

Hyperspectral remote sensors acquire a massive amount of data by obtaining many measurements, not knowing which data are relevant for a given problem. The trend for hyperspectral imagery is to record hundreds of channels from the same scene. The obtained data can characterize the chemical composition of different materials and potentially be helpful in analyzing different objects of interest.

In the spectral domain, each spectral channel is considered as one dimension and each pixel is represented as a point in that domain. By increasing the number of spectral channels in the spectral domain, theoretical and practical problems may arise and conventional techniques which are applied on multispectral data are no longer appropriate for the processing of high dimensional data [1-3].

The aforementioned characteristics show that conventional techniques based on the computation of the fully dimensional

P. Ghamisi and J. A. Benediktsson are with the Faculty of Electrical and Computer Engineering, University of Iceland, 107 Reykjavik, Iceland (corresponding author, e-mail: peg6@hi.is)

M. S. Couceiro is with Ingeniarius, Lda., and the Polytechnic Institute of Coimbra, 3030-199, Coimbra, Portugal.

This research was supported in part by the Icelandic Research Fund for Graduate Students. space, may not provide accurate classification results when the number of training samples is not substantial. For instance, while keeping the number of samples constant, after a few number of bands, the classification accuracy actually decreases as the number of features increases [1]. For the purpose of classification, these problems are related to the curse of dimensionality [4]. In order to tackle this issue and use a smaller number of training samples, the use of feature selection and extraction techniques would be of importance.

From one point of view, feature selection techniques can be split into two categories: Unsupervised and supervised. Supervised feature selection techniques aim at finding the most informative features with respect to the available prior knowledge and lead to better identification and classification of different classes of interest. On the contrary, unsupervised methods are used in order to find distinctive bands when a prior knowledge of the classes of interest is not available. Information Entropy [5], First Spectral Derivative [6] and Uniform Spectral Spacing [7] can be considered as unsupervised feature selection techniques, while supervised feature selection techniques usually try to find a group of bands achieving the largest class separability. Class separability can be calculated by considering several approaches such as Divergence [8], Transformed divergence [8], Bhattacharyya distance [9] and Jeffries-Matusita distance [8]. A comprehensive overview of different feature selection and extraction techniques is provided in [10]. However, these metrics usually suffer from the following shortcomings:

1) They are usually based on the estimation of the second order statistics (e.g., covariance matrix) and in this case, they demand many training samples in order to estimate the statistics accurately. Therefore, in a situation when the number of training samples is limited, that may lead to the singularity of the covariance matrix. In addition, since the bands in hyperspectral data usually have some redundancy, the probability of the singularity will even increase.

2) In order to select informative bands, corrupted bands (e. g., water absorption bands and bands with a low SNR), are usually pre-removed, which is a time-consuming task. Furthermore, conventional feature selection methods can be computationally demanding. To select an $m$ feature subset out of a total of $n$ features, $n ! /(n-m) ! m$ ! operations must be calculated, which is a laborious task and demands a significant amount of computational memory. In other words, the conventional feature se- 
lection techniques are only feasible in relatively low dimensional cases.

In order to address the above-mentioned shortcomings of the conventional feature selection techniques, the use of stochastic and bio-inspired optimization based feature selection techniques (e.g., Genetic Algorithms (GA) and Particle Swarm Optimization (PSO)) are considered attractive. The main reasons behind this trend is that 1) in evolutionary feature selection techniques, there is no need to calculate all possible alternatives in order to find the most informative bands and furthermore, 2) in the evolutionary approaches, usually a metric is chosen as the fitness function which is not based on the calculation of the second order statistics, and, therefore, the singularity of the covariance matrix is not a problem. In the literature, there is an extensive number of works related to the use of evolutionary optimization based feature selection techniques. These methods are mostly based on the use of GA and PSO. For example, in [11], the authors proposed a SVM classification system which allows the detection of the most distinctive features and the estimation of the SVM parameters (e.g., regularization and kernel parameters) by using a GA. In [12], authors proposed to use PSO in order to select the most informative features obtained by morphological profiles for classification. In [13], in order to address the main shortcomings of GA- and PSO-based feature selection techniques and to take the advantage of their strength, a new feature selection approach is proposed, which is based on the hybridization of GA and PSO. In [14], a method was introduced which allows to simultaneously solve problems of clustering, feature detection, and class number estimation in an unsupervised way. However, this method suffers from the computational time required by the optimization process.

In GA, if a chromosome is not selected for mating, the information contained by that individual is lost, since the algorithm does not have a memory of its previous behaviors. Furthermore, PSO suffers from the premature convergence of a swarm, because 1) particles try to converge to a single point, which is located on a line between the global best and the personal best positions, which this point does not guarantee to be a local optimum [15] and 2) furthermore, the fast rate of information flow between particles, can lead to the creation of similar particles. This results in a loss in diversity [16].

In this paper, a novel feature selection approach is proposed which is based on a new binary optimization technique and the SVM classifier. The new approach is capable of handling very high dimensional data even when only a limited number of training samples is available (ill-posed situation) and when conventional techniques are not able to proceed. In addition, despite the conventional feature selection techniques for which the number of desired features need to be initialized by the user, the proposed approach is able to automatically select the most informative features in terms of classification accuracy within an acceptable CPU processing time without requiring the number of desired features to be set a priori by users. The new feature selection technique is, at first, compared with the traditional PSO-based feature selection in terms of classification accuracy on validation samples and CPU processing time. Then, the new method is compared with a few well-known feature selection and extraction techniques. Furthermore, the new method will be taken into account in order to overcome the main shortcomings of using attribute profiles.

The rest of this paper is organized as follows: First, the new feature selection approach is described in Section II. Then, Section III briefly describes support vector machine. Section IV is devoted to the methodology of the proposed approach. Section $\mathrm{V}$ is on experimental results and main conclusion remarks are furnished in section VI.

\section{Fractional Order Darwinian Particle SWARM OPTIMIZATION (FODPSO) BASED FEATURE SELECTION}

In brief, the goal is to overcome the curse of dimensionality [4] by selecting the optimal $l_{E}$ bands for the classification, $l_{E} \leq l$, wherein $l$ is the total number of bands in a given image $I$. Selecting the most adequate bands is a complex task as the classification Overall Accuracy (OA) first grows and then declines as the number of spectral bands increases [4]. Hence, this paper tries to find the optimal $l_{E}$ bands that maximize the OA obtained as:

$$
O A=\frac{\sum_{i}^{N_{c}} C_{i i}}{\sum_{i}^{N_{c}} C_{i j}} \times 100,
$$

wherein $C_{i j}$ is the number of pixels assigned to the class $j$ which belongs to class $i . C_{i i}$ denotes the number of pixels correctly assigned to the class $i$ and $N_{c}$ is the number of classes.

In this paper, optimal features are selected through an optimization procedure in such a way that each solution gets its fitness value from the SVM classifier over validation samples. The optimization procedure is handled with PSO algorithms.

In 1995, Eberhart and Kennedy proposed the PSO algorithm for the first time [17]. The stochastic optimization ability of the algorithm is enhanced due to its cooperative simplistic mechanism, wherein each particle presents itself as a possible solution of the problem, e.g., the best $l_{E}$ bands. These particles travel through the search space to find an optimal solution, by interacting and sharing information with other particles, namely their individual best solution (personal best) and computing the global best [18].

The success of this algorithm gave rise to a chain of PSO-based alternatives in recent years, so as to overcome its drawbacks, namely, the stagnation of particles around suboptimal solutions. One of the proposed methods was denoted as Darwinian PSO (DPSO) [19]. The idea is to run many simultaneous parallel PSO algorithms, each one as a different swarm, on the same test problem and then a simple natural selection mechanism is applied. When a search tends to a suboptimal solution, the search in that area is simply discarded and another area is searched instead. In this approach, at each step, swarms that get better are rewarded (extend particle life or spawn a new descendent) and swarms that stagnate are punished (reduce swarm life or delete particles). For more information regarding how these rewards and punishments can be applied, please see [20]. DPSO has been investigated for the segmentation of remote sensing data in [21]. 
Despite the positive results retrieved by Tillett et al. [19], this coopetitive approach also increases the computational complexity of the optimization method. As many swarms of cooperative test solutions (i.e., particles) run simultaneously in a competitive fashion, the computational requirements increase and, as a consequence, the convergence time also increases. Therefore, and to further improve the DPSO algorithm, an extended version denoted as Fractional Order DPSO (FODPSO) was presented in [22], in which fractional calculus is used to control the convergence rate of the algorithm. This method has been further investigated for gray scale and hyperspectral image segmentation in [20,23]. An important property revealed by fractional calculus is that, while an integer-order derivative just implies a finite series, the fractional-order derivative requires an infinite number of terms. In other words, integer derivatives are "local" operators, while fractional derivatives have, implicitly, a "memory" of all past events. The characteristics revealed by fractional calculus make this mathematical tool well suited to describe phenomena, such as the dynamic phenomena of particles' trajectories.

Therefore, supported on the FODPSO previously presented in [22], and based on the Grunwald Letnikov definition of fractional calculus, in each step $t$, the fitness function represented by (1) is used to evaluate the success of particles (i.e., OA). To model the swarm, each particle $n$ moves in a multidimensional space according to the position $\left(x_{n}[t]\right)$, and velocity $\left(v_{n}[t]\right)$, values which are highly dependent on local best $\left(\breve{x}_{n}[t]\right)$ and global best $\left(\breve{g}_{n}[t]\right)$ information:

$$
\begin{aligned}
& v_{n}^{s}[t+1]= \\
& w_{n}^{s}[t+1]+\rho_{1} r_{1}\left(\breve{g}_{n}^{s}[t]-x_{n}^{s}[t]\right)+\rho_{2} r_{2}\left(\breve{x}_{n}^{s}[t]-x_{n}^{s}[t]\right), \\
& w_{n}^{s}[t+1]= \\
& \alpha v_{n}^{s}[t]+\frac{1}{2} \alpha(1-\alpha) v_{n}^{s}[t-1]+\frac{1}{6} \alpha(1-\alpha)(2-\alpha) v_{n}^{s}[t-2] \\
& +\frac{1}{24} \alpha(1-\alpha)(2-\alpha)(3-\alpha) v_{n}^{s}[t-3] .
\end{aligned}
$$

Since the proposed FODPSO based feature selection approach is based on running many simultaneous swarms in parallel over the search space, ' $s$ ' shows the number of each swarm.

The coefficients $\rho_{1}$ and $\rho_{2}$ are assigned weights, which control the inertial influence of "the globally best" and "the locally best", respectively, when the new velocity is determined. Typically, $\rho_{1}$ and $\rho_{2}$ are constant integer values, which represent "cognitive" and "social" components with $\rho_{1}+\rho_{2}<2$ [24]. However, different results can be obtained by assigning different values for each component.

The fractional coefficient $\alpha$, will weigh the influence of past events on determining a new velocity, $0<\alpha<1$. With a small $\alpha$, particles ignore their previous activities, thus ignoring the system dynamics and becoming susceptible to get stuck in local solutions (i.e., exploitation behavior). On the other hand, with a large $\alpha$, particles will have a more diversified behavior, which allows exploration of new solutions and improves the long-term performance (i.e., exploration behavior). However, if the exploration level is too high, then the algorithm may take longer to find the global solution. Based on [24], a good $\alpha$ value can be selected in the range of 0.6 to 0.8 .

The parameters $r_{1}$ and $r_{2}$ are random vectors with each component generally a uniform random number between 0 and 1.

In order to investigate FODPSO for the purpose of feature selection, the dimension of each particle should be equal to the number of features. In this way, the velocity dimension $\left(\operatorname{dim} v_{n}[t]\right)$, as well as the position dimension $\left(\operatorname{dim} x_{n}[t]\right)$, correspond to the total number of bands of the image, i.e., dim $v_{n}[t]=\operatorname{dim} x_{n}[t]=l$. In other words, each particle's velocity will be represented as a $l$-dimension vector. In addition, as one wishes to use the algorithm for band selection, each particle represents its position in binary values, i.e., 0 or 1 , which 0 demonstrates the absence of the corresponding feature and 1 has a dual meaning. In this case, as proposed by Khanesar et al. [25], the velocity of a particle can be associated to the probability of changing its state as:

$$
\Delta x_{n}[t+1]=\frac{1}{1+e^{-v_{n}[t+1]}}
$$

Nevertheless, as one wishes to use the algorithm for band selection, each particle represents its position in binary values, i.e., 0 or 1 . This may be represented as:

$$
x_{n}[t+1]=\left\{\begin{array}{l}
1, \Delta x_{n}[t+1] \geq r_{x} \\
0, \Delta x_{n}[t+1]<r_{x}
\end{array}\right.
$$

wherein $r_{x}$ is a random $l$-dimension vector with each component generally a uniform random number between 0 and 1 . Therefore, each particle moves in a multidimensional space according to position $x_{n}[t]$ from the discrete time system represented by equations (2), (3), (4) and (5). In other words, each particle's position will be represented as a $l$-dimensional binary vector.

To make it easier to understand of the proposed strategy, an example is given in Fig. 1. As the figure shows, the image has only 5 bands, i.e., $l=5$. This means that each particle will be defined by its current velocity and position in the 5dimensional space, i.e., $\operatorname{dim} v_{n}[t]=\operatorname{dim} x_{n}[t]=5$. In this example, and to allow a straightforward understanding, only a swarm of two particles was considered. As it is possible to observe at time/iteration $t=1$, particle 1 is positioned in such a

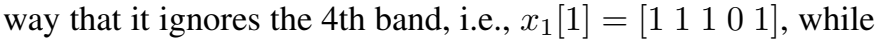
particle 2 ignores the 1st and 3rd bands i.e., $x_{2}[1]=\left[\begin{array}{llll}0 & 1 & 0 & 1\end{array}\right]$. Computing (1) under those conditions returns an overall accuracy of $O A_{1}=60 \%$ and $O A_{2}=64 \%$ for particles 1 and 2, respectively. Considering only those two particles, particle 2 will be considered as the best performing one from the swarm, thus attracting particle 1 toward it. Such attraction will induce the velocity of particle 1 for iteration 2 and, consequently, its position. ${ }^{1}$

\section{SUPPORT Vector MAchine (SVM)}

As discussed before, in the proposed method, the OA of SVM over validation samples is considered as the fitness

\footnotetext{
${ }^{1}$ The MATLAB code for the PSO- and FODPSO-based feature selection approaches will be provided on a request by sending an email to the authors.
} 


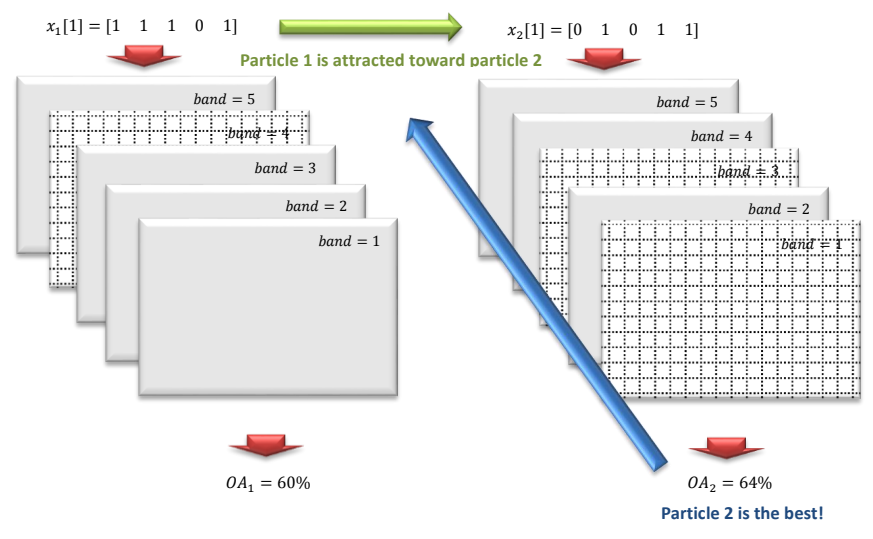

Fig. 1. Band coding of two particles for an image with 5 bands. Gridded bands are ignored in the classification process.

value. SVM has attracted much attention due to its capability of handling the curse of dimensionality in comparison with conventional classification techniques. The main reasons behind success of the approach are 1) SVM is based on a margin maximization principle which helps it to avoid estimating the statistical distributions of different classes in the hyperdimensional feature space and 2) SVM takes advantage of the strong generalization capability obtained by its sparse representation of the decision function [11].

In hyperspectral image analysis, the Random Forest (RF) classifier and SVM play a key role since they can handle high dimensional data even with a limited number of training samples. In this work, we prefer to use SVM rather than RF due to its susceptibility to noise. Due to its sensibility, corrupted and noisy bands may significantly influence on the classification accuracies. As a result, when RF is considered as the fitness function, due to its capability to handle different types of noises, corrupted bands cannot be eliminated even after high number of iterations. On the contrary, since SVM is more sensible than RF against noises, SVM can detect and eliminate corrupted bands after a few iterations, which can be considered as a privilege for the final classification step.

The general idea behind SVM is to separate training samples belonging to different classes by tracing maximum margin hyperplanes in the space where the samples are mapped [26]. SVMs were originally introduced for solving linear classification problems. However, they can be generalized to nonlinear decision functions by considering the so-called kernel trick [27]. A kernel-based SVM is being used to project the pixel vectors into a higher dimensional space and estimate maximum margin hyperplanes in this new space, in order to improve linear separability of data [27]. The sensitivity to the choice of the kernel and regularization parameters can be considered as the most important disadvantages of SVM. The latter is classically overcome by considering cross-validation techniques using training data [28]. The Gaussian radial basis function $(\mathrm{RBF})$ is widely used in remote sensing [27]. More information regarding SVM can be found in [29, 30].

\section{METHODOLOGY}

In order to show the different capabilities of the proposed feature selection technique, two different scenarios have been taken into consideration.

\section{A. First scenario}

In the first scenario, the new feature selection approach is directly performed on raw data sets in order to select the most informative bands from the whole data set. The main work flow of the proposed method for this scenario is listed below:

1) Training samples are split into two categories: Training and validation samples.

2) FODPSO-based feature selection is performed on the raw data. SVM is chosen as the fitness function and its corresponding OA is considered as the fitness value.

3) The selected bands are classified by SVM with the whole training and test samples and final classification map will be achieved.

\section{B. Second scenario}

In the second scenario, an application of the proposed FODPSO-based feature selection technique will be shown. In this scenario, we address the main shortcomings of using Attribute Profile (AP): 1) which attributes should be taken into account and 2) which values should be opted as threshold values. A comprehensive discussion related to AP and its all modifications and alternatives, can be found in [31]. In this scenario, different types of attributes with the wide ranges of threshold values will be constructed for building a feature bank and then, we let the proposed feature selection technique choose the most informative features from the bank with respect to the classification accuracy for the validation samples. In other words, the new feature selection technique not only solves the main shortcomings associated with the concept of AP, but also reduces the redundancy of the features and addresses the curse of dimensionality.

Fig. 2 illustrates the flowchart of the proposed method based on FODPSO feature selection technique for the second scenario. The main work flow of this method is listed below:

1) A feature bank is made, consisting of raw input data and an AP obtained with four attributes with a wide range of threshold values.

- The raw input data are transformed by Principal Component Analysis (PCA).

- The most important Principal Components (PCs), i.e., components with cumulative variance of more than $99 \%$, are kept and used as base images for the Extended Multi-AP (EMAP).

- The obtained EMAP and the raw input data are concatenated into a stacked vector (let us call the output of this step $\wp$ ).

2) Training samples are split into two categories: Training and validation samples.

3) FODPSO based feature selection is performed on $\wp$. The fitness of each particle is evaluated by the OA of SVM for the validation samples. After a few iterations, 


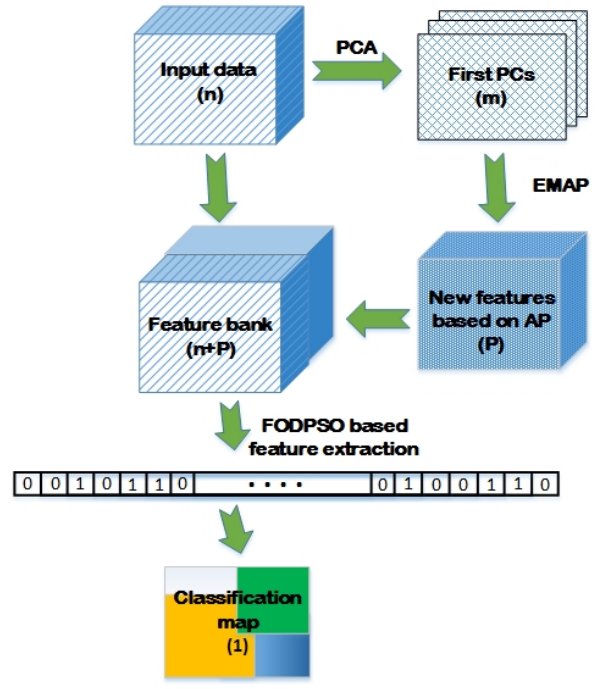

Fig. 2. General idea of the proposed classification framework based on FODPSO-based feature selection technique for the second scenario.

the FODPSO based feature selection approach finds the most informative bands with respect to the OA of SVM over the validation samples (the output of this step will be called $\Im$ ).

4) $\Im$ is classified by SVM by considering the whole set of training and test samples and a final classification map will be achieved.

It should be noted that, in this work, the PCA can be replaced by other feature extraction techniques (in particular Kernel PCA and NWFE which have shown promising results in order to produce attribute profiles [31]). Now, a brief discussion on AP is given.

1) Extended Multi-Attribute Profile (EMAP): In order to overcome the shortcomings of the morphological profile, AP was introduced in [32] for extracting spatial information of the input data, which is based on attribute filters. APs can be regarded as more effective filters than morphological profiles because the concept of the attribute filters is not limited to only the size of different objects and the APs are able to characterize other characteristics such as shape of existing objects in the scene. In addition, APs are computed according to an effective implementation based on max-tree and min-tree representations, which lead to a reduction of the computational load when compared with conventional profiles built with operators by reconstruction. An AP is built by the sequence of attribute thinning and thickening transformations defined with a sequence of progressively stricter criteria [32]. To handle hyperspectral images, the extension of AP was proposed in [33]. Extended AP (EAP) is a stacked vector of different APs computed on the first $C$ features extracted from the original data set. When different attributes, $a_{1}, a_{2}, \ldots, a_{M}$ are concatenated into a stacked vector, the EMAP is obtained. More information regarding AP and its different variations can be found in $[2,3,31,32]$. In this paper, the following attributes have been taken into account:

1) (a) area of the region (related the size of the regions),

2) (s) standard deviation (as an index for showing the

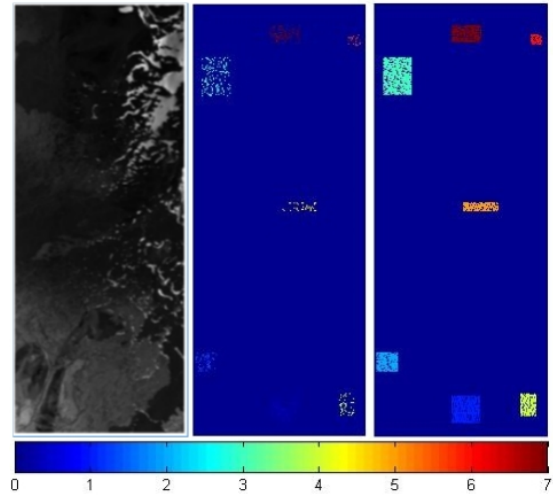

Fig. 3. The AVIRIS Hekla data set. a) Spectral band number 50; b) training samples, c) test samples, where each color represents a specific information class. The information classes are listed in Table I.

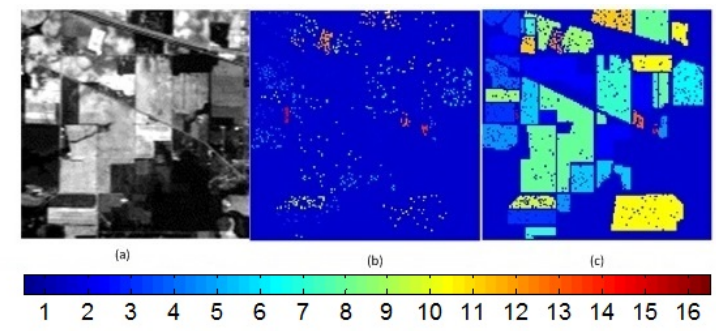

Fig. 4. The AVIRIS Indian Pines data set. a) Spectral band number $27(\lambda=$ $646.72 \mu)$; b) training samples, c) test samples, where each color represents a specific information class. The information classes are listed in Table II.

homogeneity of the regions),

3) (d) diagonal of the box bounding the regions,

4) (i) moment of inertia (as an index for measuring the elongation of the regions).

\section{EXPERIMENTAL RESULTS}

\section{A. Data Description}

1) Hekla data: The first hyperspectral data set used was collected on June 17, 1991 by AVIRIS (having spatial resolution $20 \mathrm{~m}$ ) from the volcano Hekla in Iceland (see Fig. 3). Sixty four bands (from $1.84 \mu$ to $2.4 \mu$ ) were removed due to the technical problem with the 4th spectrometer in 157 bands. An image of size $500 \times 200$ was used in this paper for the real experiments. For this data set, from the total number of training samples which is equal to 966,50 percent was chosen for training and the rest as validation samples, in order to perform the PSO- and FODPSO-based feature selection approaches. After finding the most informative bands with respect to the OA of SVM over validation samples, all 966 samples are used for training in order to perform SVM on the selected bands. The number of training, validation and test samples are displayed in Table I.

2) Indian Pines data: The second data set used in experiments is the well-known data set captured on Indian Pines (NW Indiana) in 1992 comprising 16 classes (see Fig. 4), mostly related to different land covers. The data set consists of $145 \times 145$ pixels with spatial resolution of $20 \mathrm{~m} /$ pixel. In this 
TABLE I

HEKLA: NUMBER OF TRAINING, VALIDATION AND TEST SAMPLES. FOR THE FINAL CLASSIFICATION STEP, THE TOTAL OF TRAINING AND VALIDATION SAMPLES IS USED TO TRAIN THE SVM.

\begin{tabular}{ccccc}
\hline \multirow{2}{*}{ Number } & Class & \multicolumn{3}{c}{ Number of Samples } \\
& Name & Training & Validation & Test \\
\hline 1 & Andesite lava 1970 & 97 & 97 & 829 \\
2 & Andesite lava 1980 I & 54 & 54 & 442 \\
3 & Andesite lava 1991 I & 150 & 150 & 1196 \\
4 & Andesite lava moss cover & 49 & 49 & 370 \\
5 & Hyaloclastite formation & 38 & 38 & 334 \\
6 & Rhyolite & 20 & 19 & 143 \\
7 & Firn-glacier ice & 76 & 75 & 549 \\
\hline
\end{tabular}

TABLE II

INDIAN PINES: NUMBER OF TRAINING, VALIDATION AND TEST SAMPLES. FOR THE FINAL CLASSIFICATION STEP, THE TOTAL OF TRAINING AND VALIDATION SAMPLES IS USED TO TRAIN THE SVM.

\begin{tabular}{ccccc}
\hline & Class & \multicolumn{3}{c}{ Number of Samples } \\
Number & Name & Training & Validation & Test \\
\hline 1 & Corn-notill & 25 & 25 & 1384 \\
2 & Corn-mintill & 25 & 25 & 784 \\
3 & Corn & 25 & 25 & 184 \\
4 & Grass-pasture & 25 & 25 & 447 \\
5 & Grass-trees & 25 & 25 & 697 \\
6 & Hay-windrowed & 25 & 25 & 439 \\
7 & Soybean-notill & 25 & 25 & 918 \\
8 & Soybean-mintill & 25 & 25 & 2418 \\
9 & Soybean-clean & 25 & 25 & 564 \\
10 & Wheat & 25 & 25 & 162 \\
11 & Woods & 25 & 25 & 1244 \\
12 & Bldg-Grass-Tree-Drives & 25 & 25 & 330 \\
13 & Stone-Steel-Towers & 25 & 25 & 45 \\
14 & Alfalfa & 8 & 7 & 39 \\
15 & Grass-pasture-mowed & 8 & 7 & 11 \\
16 & Oats & 8 & 7 & 5 \\
\hline
\end{tabular}

work, 220 data channels (including all noisy and atmospheric absorbed bands) are used. In the same way, for Indian Pines, from the total number of training samples which is equal to 695,50 percent of the samples were chosen for training and the rest for the validation samples, in order to perform the PSO- and FODPSO-based feature selection approaches. After performing PSO- and FODPSO-based feature selection approaches, all 695 samples are used for training in order to perform SVM on the selected bands. The number of training, validation and test samples are displayed in Table II.

In this work, in addition to selecting data sets that are widely used in the hyperspectral imaging community, we have used exactly the same training and test samples that have been considered in most works related to the classification of hyperspectral images. Some of the works which have considered exactly the same training and test samples are given in references [3, 34]. In other words, we not only used the same number of training and test samples adopted by other state-ofthe-art methods, but the samples also have exactly the same spatial locations in the data. This way of using the training and test samples makes this work fully comparable with other spectral and spatial classification techniques reported in the literature.

\section{B. General Information}

The following measures are used in order to evaluate the performance of different classification methods.

1) Average Accuracy ( $A A)$ : This index shows the average value of the class classification accuracy.

2) Overall Accuracy (OA): This index represent the number of samples which is classified correctly divided by the number of test samples.

3) Kappa Coefficient ( $k$ ): This index provides information regarding the amount of agreement corrected by the level of agreement that could be expected due to chance alone.

4) CPU Processing Time: This measure shows the speed of different algorithms. It should be noted, since in all algorithms (except Raw), EMAP is carried out, the CPU processing time of this step is discarded from all methods. Hence, the CPU processing time is only provided for AP, Raw + AP, Decision Boundary Feature Extraction (DBFE) and NWFE. Except DBFE and NWFE, which have been used in the MultiSpec software, all methods used were programmed in MATLAB on a computer having Intel(R) Pentium(R) 4 CPU $3.20 \mathrm{GHz}$ and 4GB of memory.

The number of iterations in each run for PSO- and FODPSO-based feature selection techniques is equal to 10 . Since PSO- and FODPSO-based feature selection techniques are randomized methods which are based on different first populations, each algorithm has here been run 30 times and results are shown in different histograms and compared with different indexes in order to examine the capabilities of PSOand FODPSO-based feature selection techniques.

It should be noted that, in this paper, in order to compare PSO and FODPSO, both data sets (Hekla and Indian Pines) have been taken into account. However for the first and second scenarios, we preferred to use only Indian Pines since this data set is more complex for classification. Therefore, the capability of the proposed method can be shown more clearly by using Indian Pines instead of Hekla. In this case, 25 PCs with a cumulative variance of more than $99 \%$ were selected as the base images for producing EMAP for Indian Pines.

Based on [24], the sum of all $\rho$ 's should be inferior to 2 and alpha should be near 0.632 . Therefore, the parameters $\rho_{1}, \rho_{2}$ and $\alpha$ are initialized by $0.8,0.8$ and 0.7 , respectively. It should be noted that, the same set of parameters have been used for both data sets and both scenarios, in order to show that the proposed technique is data set distribution independent, and with the same set of parameters, for all different data sets and scenarios, the proposed method can lead to an acceptable results in terms of accuracies and CPU processing time.

After comparing PSO- and FODPSO-based feature selection techniques in terms of overall accuracy over validation samples, the best method will be chosen for further evaluation. Then, the best method will be compared with the other wellknown feature selection and extraction techniques. In order to have a fair comparison, among 30 runs, four runs have been chosen and their classification accuracies are compared with obtained results from the other feature selection and extraction techniques. In this way, the results of 30 runs have been sorted in an increasing order with respect to their overall accuracy 
over validation samples. Then, for a fair comparison with the other methods, the four runs are selected as follows:

1) Min: SVM classification is applied on the bands selected with the least overall accuracy over the validation samples among 30 runs (the first group of the most informative bands when the results of 30 runs have been sorted in an increasing order).

2) Median ${ }^{1}:$ SVM classification is applied on the bands selected with the median overall accuracy over the validation samples among 30 runs (the 15th group of the most informative bands when the results of 30 runs have been sorted in an increasing order).

3) Median ${ }^{2}$ : SVM classification is applied on the the most informative bands selected with the median overall accuracy over the validation samples among 30 runs (the 16th group of bands when the results of 30 runs have been sorted in an increasing order).

4) Max: SVM classification is applied on the bands selected with the highest overall accuracy over the alidation samples among 30 runs (the 30th group of the most informative bands when the results of 30 runs have been sorted in an increasing order).

Other methods for the purpose of comparison are listed below:

1) Raw: The input data are directly classified with SVM without performing any feature selection or extraction technique.

2) Div: Divergence feature selection is performed on the input data and the selected bands are classified by SVM.

3) TD: Transformed Divergence feature selection is performed on the input data and the selected bands are classified by SVM.

4) Bhathacharyya: Bhathacharyya distance feature selection is performed on the input data and the selected bands are classified by SVM.

5) $D B F E$ : DBFE is performed on the input data and the selected bands are classified by SVM.

6) NWFE: NWFE is performed on the input data and the selected bands are classified by SVM,

and for the second scenario,

1) $A P$ : The feature bank including all four attributes with a wide range of threshold values is classified by SVM.

2) Raw $+A P$ : The Raw and $A P$ are concatenated into a stacked vector and classified by SVM.

The following ranges for different attributes have been taken into account in order to build the feature bank:

$a=\left(\frac{1000}{p h i}\right) \times\{1,2,3,4,5,6,7,8,9,10,11,12,13,14\}$

$s=\left(\frac{\mu}{100}\right) \times\{5,10,15,20,25,30,35,40,45,50\}$

$d=\{10,20,30,40,50,60,70,80,90,100\}$

$i=\{0.1,0.2,0.3,0.4,0.5,0.6,0.7,0.8,0.9\}$

where phi and $\mu$ are the resolution of the image in meters and the mean of a feature, respectively.

Again, in order to secure the fairness of the comparison, the number of features for DBFE and NWFE has been chosen in 2 different ways: 1) the number of selected features is equal to the number of features which provides Max (see a few lines above) and 2) the top few eigenvalues which account for $99 \%$ of the total sum of the eigenvalues were selected.

The data sets have been classified with SVM and a Gaussian kernel. Five-fold cross validation is taken into account in order to select the hyperplane parameters when SVM is used for the last step (for the classification of informative bands).

\section{FODPSO vs. PSO}
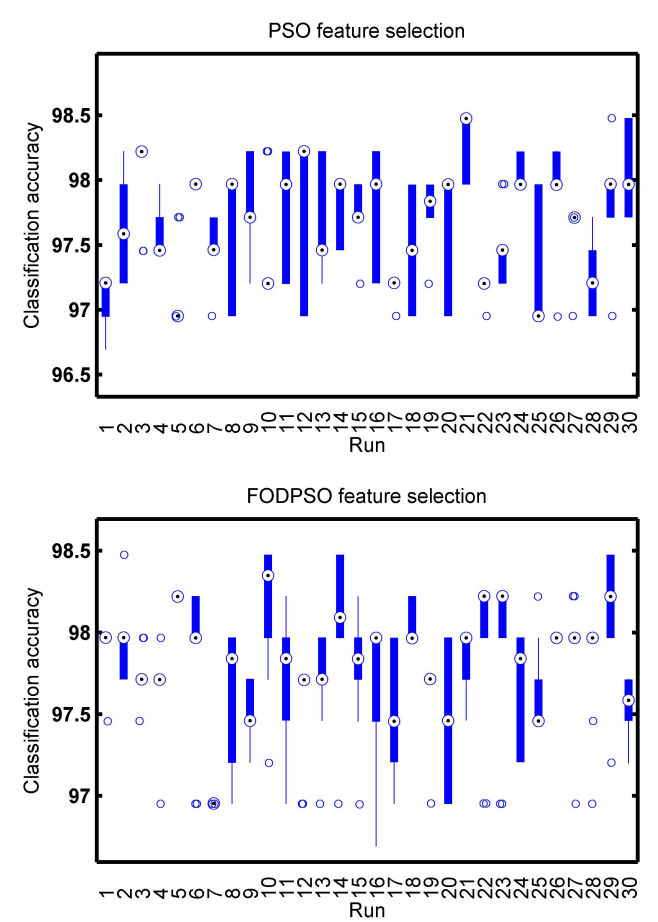

Fig. 5. Hekla: Box plots for overall accuracy over 30 runs for PSO-based feature selection (top) and FODPSO-based feature selection (bottom).

1) Hekla: Fig. 5 shows the box plots for the OA of the classification for 30 runs (10 iterations within each run) for PSOand FODPSO-based feature selection approaches, respectively. As can be observed from Fig. 5, although one cannot perceive any major differences between both methods, the FODPSObased feature selection approach presents an overall smaller interquartile range, i.e., the $\mathrm{OA}$ of the classification, at each run, has less dispersion regardless of the number of trials. On the other hand, the average value of the OA is slightly larger than for the PSO-based method.

One-way MANOVA analysis was carried out to assess whether both the PSO- and FODPSO-based algorithms have a statistically significant effect on the classification performance. The significance of the different types of algorithm used (independent variable) on the final $O A$ and the CPU processing time (dependent variables) was analyzed using one-way MANOVA after checking the assumptions of multivariate normality and homogeneity of variance/covariance, for a significance level of $5 \%$.

The assumption of normality of each of the univariate dependent variables was examined using a paired-sample Kolmogorov-Smirnov $(p-$ value $<0.05)$ [35]. Although the univariate normality of each dependent variable has not been 


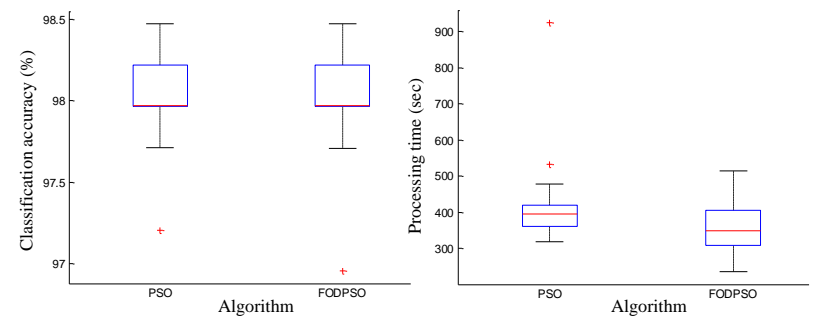

Fig. 6. Hekla: Final classification accuracy in percentage and processing time in seconds for the PSO- and FODPSO-based feature selection approaches.

verified, since $n \geq 30$ and this was assumed by benefiting from using the Central Limit Theorem (CLT) [36, 37]. Consequently, the assumption of multivariate normality was validated [37, 38]. Note that MANOVA makes the assumption that the within-group covariance matrices are equal. Therefore, the assumption about the equality and homogeneity of the covariance matrix in each group was verified with the Box's $\mathrm{M}$ Test $(M=72.7642, F(3 ; 720)=-2.0706 ; p-$ value $=$ 1.0000) [38].

The MANOVA analysis revealed that the type of algorithm did not lead to a statistically significant different outcome for the multivariate composite $(F(1 ; 58)=3.5830 ; p-$ value $=$ 0.1667). In this situation, the FODPSO-based solution produces slightly better solutions than the PSO but is considerably faster than the latter. To easily assess the differences between both algorithms, let us graphically show the outcome of each trial using box plot charts (Fig. 6). The ends of the blue boxes and the horizontal red line in between correspond to the first and third quartiles and the median values, respectively. As one may observe, by benefiting from the fractional version of the algorithm, one is able to slightly increase the OA while, at the same time, slightly decrease the CPU processing time.

2) Indian Pines: Fig. 7 shows the box plots for the OA of the classification for 30 runs (10 iterations within each run) for PSO- and FODPSO-based feature selection approaches, respectively. Similarly as before, despite the lack of major differences, the FODPSO-based feature selection approach presents an overall smaller interquartile range and larger average value of the overall accuracy.

Once again, one-way MANOVA analysis was carried out to assess whether both PSO- and FODPSO-based algorithms have a statistically significant effect on the classification performance.

The assumption about the equality and homogeneity of the covariance matrix in each group was verified with the Box's $\mathrm{M}$ Test $(M=72.5156, F(3 ; 720)=-2.0636 ; p-$ value $=$ 1.0000).

For this data set, the MANOVA analysis revealed that the type of the feature selection algorithm led to a statistically significant different outcome on the multivariate composite $(F(1 ; 58)=14.6338 ; p-$ value $<0.0001)$. As the MANOVA detected significant statistical differences, we proceeded to the commonly-used ANOVA for each dependent variable. By carrying an individual test on each dependent variable, it was possible to observe that the OA does not present statistically significant differences $(F(1 ; 58)=0.0116$;
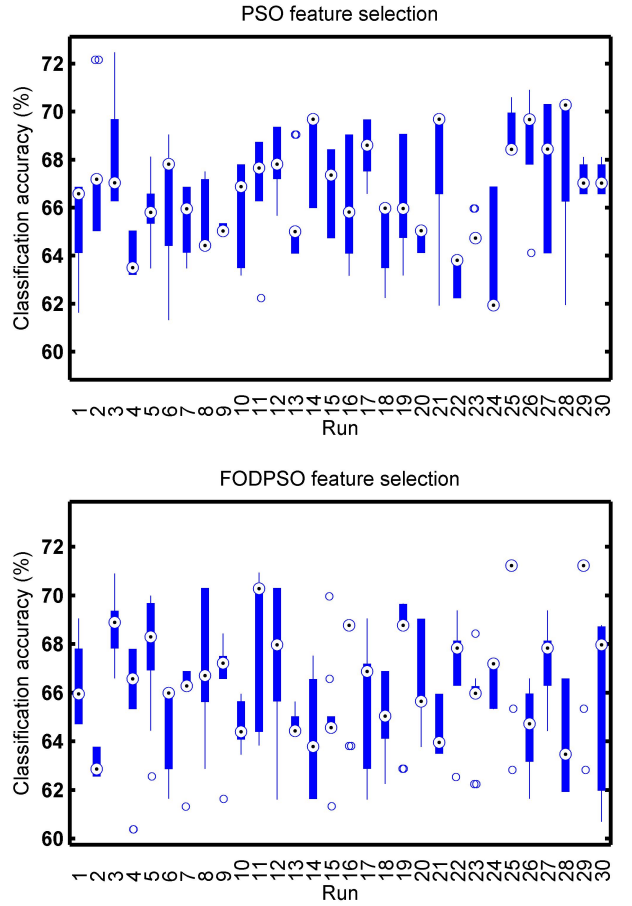

Fig. 7. Indian Pines: Box plots for overall accuracy in percentage over 30 runs for PSO-based feature selection (top) and FODPSO-based feature selection (bottom).
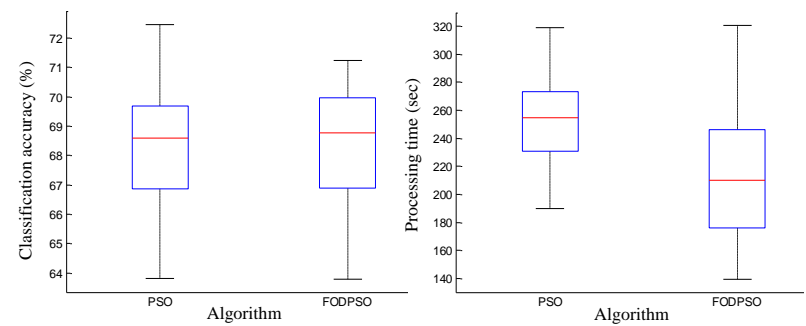

Fig. 8. Indian Pines: Final classification accuracy in percentage of the PSOand FODPSO-based feature selection approaches.

$p$ - value $=0.9145$ ). On the other hand, it is in the CPU processing time that both algorithms diverge the most, thus resulting in statistically significant differences between them $(F(1 ; 58)=16.7499 ; p-$ value $<0.0001)$. As expected, the FODPSO-based solution produces slightly better solutions than the PSO considerably faster than the latter.

To easily assess the differences between both algorithms, the outcome of each trial is shown graphically using box plot charts (Fig. 8). As one may observe, by benefiting from the fractional version of the algorithm, one is able to slightly increase the OA (slightly higher median value) while, at the same time, considerably decrease the CPU processing time.

\section{First Scenario}

Fig. 9 shows the list of the selected bands by the proposed method in 30 different runs. Runs 2, 16, 30 and 29 are selected as Min, Median ${ }^{1}$, Median ${ }^{2}$ and Max, respectively. As it was mentioned before, since the FODPSO-based feature selection technique is a randomized method which is based on different 
TABLE III

FIRST SCENARIO: THE CLASSIFICATION OF DIFFERENT TECHNIQUES IN PERCENTAGE FOR INDIAN PINES. THE NUMBER OF FEATURES IS SHOWN IN BRACKETS. THE BEST ACCURACY IN EACH ROW IS SHOWN IN BOLD.

\begin{tabular}{c|c|c|c|c|c|c|c|c|c}
\hline \hline $\begin{array}{c}\text { Class } \\
\text { No. }\end{array}$ & $\begin{array}{c}\text { Raw } \\
(220)\end{array}$ & $\begin{array}{c}\text { Min } \\
(84)\end{array}$ & $\begin{array}{c}\text { Median }^{1} \\
(79)\end{array}$ & $\begin{array}{c}\text { Median }^{2} \\
(57)\end{array}$ & $\begin{array}{c}\text { Max } \\
(44)\end{array}$ & $\begin{array}{c}\text { DBFE } \\
(44)\end{array}$ & $\begin{array}{c}\text { DBFE-99\% } \\
(17)\end{array}$ & $\begin{array}{c}\text { NWFE } \\
(44)\end{array}$ & $\begin{array}{c}\text { NWFE-99\% } \\
(120)\end{array}$ \\
\hline 1 & 54.1 & 67.1 & 69.5 & 67.1 & $\mathbf{7 0 . 9}$ & 54.4 & 54.1 & 59.6 & 49.1 \\
2 & 57.5 & 67.6 & 66.8 & $\mathbf{6 9 . 6}$ & 68.6 & 62.2 & 53.8 & 51.2 & 46.3 \\
3 & 80.4 & 82.0 & 84.2 & $\mathbf{9 0 . 7}$ & 88.5 & 73.3 & 70.1 & 67.3 & 64.6 \\
4 & 88.3 & 92.6 & 92.6 & 88.1 & $\mathbf{9 2 . 8}$ & 86.5 & 88.1 & 86.5 & 89.0 \\
5 & 81.4 & 85.2 & $\mathbf{8 8 . 5}$ & 82.6 & 88.3 & 88.3 & 87.2 & 87.5 & 84.3 \\
6 & 92.2 & 92.9 & 94.9 & 94.0 & 95.8 & 94.5 & 96.3 & $\mathbf{9 7 . 2}$ & $\mathbf{9 7 . 2}$ \\
7 & 68.0 & 73.7 & 76.6 & 76.4 & $\mathbf{7 8 . 7}$ & 61.1 & 62.2 & 61.0 & 54.0 \\
8 & 49.1 & 47.7 & $\mathbf{6 3 . 6}$ & 58.4 & 61.7 & 47.2 & 43.9 & 34.2 & 35.7 \\
9 & 64.1 & 73.7 & 82.0 & 79.7 & $\mathbf{8 3 . 5}$ & 72.3 & 67.7 & 62.2 & 63.4 \\
10 & 95.6 & 96.2 & 97.5 & $\mathbf{9 8 . 7}$ & $\mathbf{9 8 . 7}$ & $\mathbf{9 8 . 7}$ & 99.3 & $\mathbf{9 8 . 7}$ & 98.1 \\
11 & 79.0 & 82.7 & 87.1 & 87.5 & $\mathbf{9 1 . 1}$ & 86.6 & 85.3 & 80.9 & 84.4 \\
12 & 64.5 & 70 & 73.0 & 73.3 & $\mathbf{7 7 . 5}$ & 76.9 & 73.0 & 69.6 & 72.1 \\
13 & 95.5 & 97.7 & 97.7 & $\mathbf{1 0 0}$ & 97.7 & 91.1 & 93.3 & 95.5 & 91.1 \\
14 & 64.1 & 79.4 & $\mathbf{9 2 . 3}$ & 84.6 & 89.7 & 61.5 & 58.9 & 71.7 & 61.5 \\
15 & 81.8 & $\mathbf{1 0 0}$ & 81.8 & 90.9 & 90.9 & 81.8 & $\mathbf{1 0 0}$ & 81.8 & 63.6 \\
16 & $\mathbf{1 0 0}$ & $\mathbf{1 0 0}$ & $\mathbf{1 0 0}$ & 60 & $\mathbf{1 0 0}$ & 40 & 40 & 40 & 40 \\
\hline AA & 76.02 & 81.82 & 84.29 & 81.39 & $\mathbf{8 5 . 9 4}$ & 73.57 & 73.36 & 71.61 & 68.44 \\
OA & 65.41 & 70.11 & 76.22 & 74.17 & $\mathbf{7 7 . 1 8}$ & 66.95 & 64.96 & 61.98 & 60.13 \\
K & 0.6119 & 0.6646 & 0.7306 & 0.7088 & $\mathbf{0 . 7 4 1 8}$ & 0.6273 & 0.6055 & 0.5749 & 0.5533 \\
Time & 94 & 165 & 192 & 276 & 223 & 105 & 72 & 89 & 132 \\
\hline
\end{tabular}

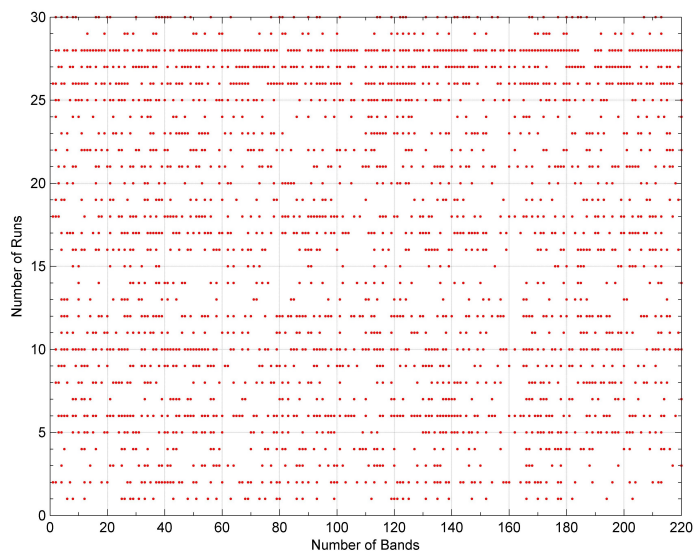

Fig. 9. First scenario: Selected bands by the proposed method in 30 different runs. Runs 2, 16, 30 and 29 are selected as Min, Median ${ }^{1}$, Median $^{2}$ and Max, respectively.

first populations, the selected bands are different in different runs.

As can be seen from Table III, the proposed method (Max) provides the best results in terms of OA, followed by Median ${ }^{1}$ and Median ${ }^{2}$ (other runs of the proposed technique). This shows that different alternatives of the proposed method (except Min) demonstrate the best performance and improve the other techniques in terms of classification accuracies.

Some algorithms, such as the originally proposed DBFE [39], require the use of the second order statistics (e.g., the covariance matrix) to characterize the distribution of training samples with respect to the mean. In hyperspectral image analysis, the number of available training samples is usually not sufficient to make a good estimate of the covariance matrix. In this case, the use of sample covariance, or common covariance [1], may not be successful. As an example, either when the sample or the common covariance approach, is chosen to estimate the statistics for each available class for DBFE, if the number of pixels in the classes is not, one more than the total number of features being used (at least), the DBFE stops working. In this case, the Leave-One-Out Covariance (LOOC) [1] estimator can be used as an alternative to estimate the covariance matrix. The normal minimum number of required samples for a sample class covariance matrix is $l+l$ samples for $l$-dimensional data. For the LOOC estimator, only a few samples are all that is needed. In general, this covariance estimator is non-singular when at least three samples are in hand regardless of the dimensions of the data, and so it can be used even though the sample covariance or common covariance estimates are singular.

As discussed before, the conventional feature selection techniques are only feasible in relatively low dimensional cases. In this way, as the number of bands increases, the required statistical estimation becomes unwieldy. In our case, the methods; Divergence, Transformed divergence and Bhattacharyya distance stopped working since in our data sets, the corrupted bands have not been eliminated and also the dimensionality of the data sets is high. However, since the proposed method is based on the evolutionary technique, there is no need to calculate all possible alternatives in order to find the most informative bands. Another advantage of using the proposed method is that there is no need to estimate the second order statistics and, in this manner, the singularity of the covariance matrix is not a problem. Therefore, the FODPSObased feature selection technique can find the most informative bands in a very reasonable CPU processing time when the other techniques stop and cannot lead to a conclusion.

\section{E. Second Scenario}

Fig. 10 depicts the box plots for the OA of the classification for 30 runs (10 iterations within each run) for PSO- and FODPSO-based feature selection approaches, respectively. As 

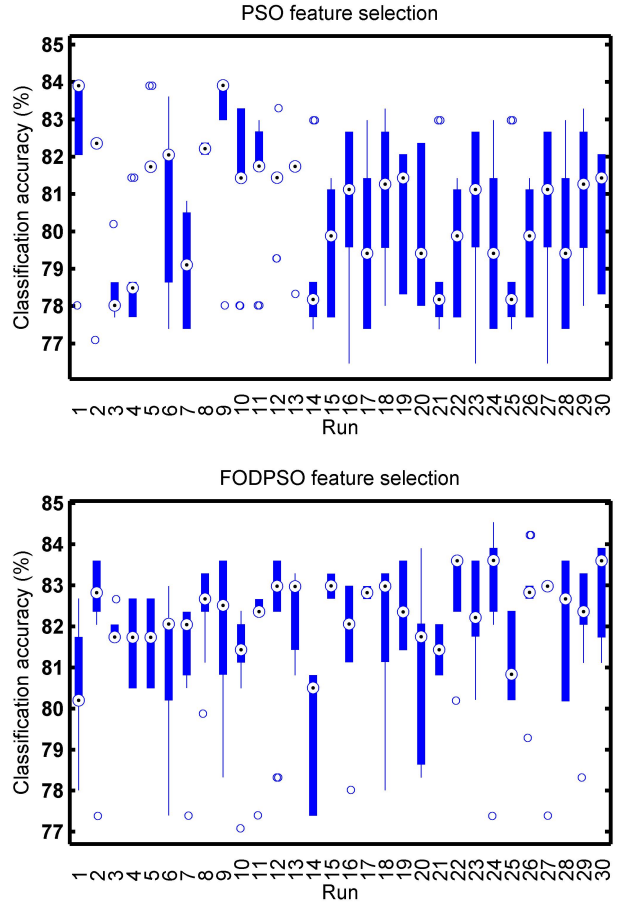

Fig. 10. Indian Pines: Box plots for overall accuracy in percentage over 30 runs for PSO-based feature selection (top) and FODPSO-based feature selection (bottom).

TABLE IV

SECOND SCENARIO: THE NUMBER OF SELECTED FEATURES IN $M i n$, Median $^{1}$, Median $^{2}$ AND Max FOR DIFFERENT ATTRIBUTES, AREA (A) WITH 725 FEATURES, STANDARD DEVIATION (S) WITH 500 FEATURES, MOMENT OF INERTIA (I) WITH 450 FEATURES AND DIAGONAL OF THE BOX BOUNDING THE REGIONS (D) WITH 500 FEATURES.

\begin{tabular}{c|ccccc|c}
\hline Group & $\mathrm{a}$ & $\mathrm{s}$ & $\mathrm{i}$ & $\mathrm{d}$ & $\begin{array}{c}\text { Input } \\
\text { data }\end{array}$ & $\begin{array}{c}\text { Total number of } \\
\text { selected features }\end{array}$ \\
\hline \hline Min & 152 & 106 & 85 & 107 & 47 & 497 \\
Median $^{1}$ & 124 & 79 & 76 & 85 & 34 & 398 \\
Median $^{2}$ & 153 & 118 & 92 & 112 & 42 & 517 \\
Max & 83 & 74 & 65 & 53 & 31 & 306 \\
\hline Total features & 725 & 500 & 450 & 500 & 220 & - \\
\hline \hline
\end{tabular}

before, Fig. 10 shows the advantage of the FODPSO-based approach over the alternative. In this case, one can easily perceive the differences, wherein both the interquartile range and average value of the OA are considerably improved. In other words, the FODPSO-based feature selection technique is able to find a better and more stable solution than the PSObased feature selection technique.

The significance of the different type of algorithm used (independent variable) on the final OA and the CPU processing time (dependent variables) was analyzed using one-way MANOVA.

The assumption about the equality and homogeneity of the covariance matrix in each group was verified with the Box's $\mathrm{M}$ Test $(M=72.8921, F(3 ; 720)=-2.07424 ; p-$ value $=$ 1.0000). This suggests that the design is balanced and, since there is an equal number of observations in each cell $(n=30)$, the robustness of the MANOVA tests is guaranteed.
TABLE $\mathrm{V}$

SECOND SCENARIO: THE CLASSIFICATION OF DIFFERENT TECHNIQUES IN PERCENTAGE FOR INDIAN PINES. THE NUMBER OF FEATURES IS SHOWN IN BRACKETS. THE BEST ACCURACY IN EACH ROW IS SHOWN IN BOLD.

\begin{tabular}{c|c|c|c|c|c|c|c}
\hline \hline $\begin{array}{c}\text { Class } \\
\text { No. }\end{array}$ & $\begin{array}{c}\text { Raw } \\
(220)\end{array}$ & $\begin{array}{c}\text { AP } \\
(2175)\end{array}$ & $\begin{array}{c}\text { Raw+AP } \\
(2395)\end{array}$ & $\begin{array}{c}\text { Min } \\
(497)\end{array}$ & $\begin{array}{c}\text { Median }^{1} \\
(398)\end{array}$ & $\begin{array}{c}\text { Median }^{2} \\
(517)\end{array}$ & $\begin{array}{c}\text { Max } \\
(306)\end{array}$ \\
\hline 1 & 54.1 & 68.3 & 67.7 & $\mathbf{7 8 . 1}$ & 76.0 & 76.3 & 76.6 \\
2 & 57.5 & 79.3 & 78.5 & 88.2 & $\mathbf{9 0 . 1}$ & 87.7 & 89.5 \\
3 & 80.4 & 82.6 & 82.6 & 94.0 & 92.9 & $\mathbf{9 5 . 6}$ & 93.4 \\
4 & 88.3 & 83.4 & 81.8 & 93.9 & $\mathbf{9 4 . 4}$ & $\mathbf{9 4 . 4}$ & $\mathbf{9 4 . 4}$ \\
5 & 81.4 & 81.2 & 80.4 & 90.2 & 90.2 & 90.1 & $\mathbf{9 0 . 3}$ \\
6 & 92.2 & 94.9 & 94.5 & 98.8 & 98.8 & 98.6 & $\mathbf{9 9 . 0}$ \\
7 & 68.0 & 75.8 & 75.8 & 82.7 & 82.0 & $\mathbf{8 4 . 8}$ & 77.4 \\
8 & 49.1 & 68.5 & 67.7 & 84.9 & 78.4 & $\mathbf{8 5 . 1}$ & 82.4 \\
9 & 64.1 & 75.5 & 73.7 & 87.2 & 84.5 & 86.8 & $\mathbf{8 9 . 1}$ \\
10 & 95.6 & 91.9 & 90.7 & 98.7 & 98.1 & $\mathbf{9 9 . 3}$ & 98.7 \\
11 & 79.1 & 88.2 & 87.9 & 95.8 & 95.5 & 94.2 & $\mathbf{9 5 . 9}$ \\
12 & 64.5 & 97.8 & $\mathbf{9 8 . 1}$ & 94.5 & 93.0 & 94.8 & 94.2 \\
13 & 95.5 & 88.8 & 88.8 & $\mathbf{1 0 0}$ & $\mathbf{1 0 0}$ & $\mathbf{1 0 0}$ & $\mathbf{1 0 0}$ \\
14 & 64.1 & 51.2 & 43.5 & 92.3 & 89.7 & 89.7 & $\mathbf{9 7 . 4}$ \\
15 & 81.8 & 81.8 & 81.8 & 81.8 & 90.9 & 90.9 & $\mathbf{1 0 0}$ \\
16 & $\mathbf{1 0 0}$ & $\mathbf{1 0 0}$ & 80 & $\mathbf{1 0 0}$ & $\mathbf{1 0 0}$ & $\mathbf{1 0 0}$ & $\mathbf{1 0 0}$ \\
\hline AA & 76.02 & 81.87 & 79.64 & 91.35 & 90.94 & 91.80 & $\mathbf{9 2 . 4 4}$ \\
OA & 65.41 & 77.54 & 76.85 & $\mathbf{8 7 . 8 3}$ & 85.73 & 87.60 & 86.78 \\
K & 0.611 & 0.7465 & 0.739 & $\mathbf{0 . 8 6 1 3}$ & 0.8377 & 0.8587 & 0.8494 \\
Time & - & 1295 & 1434 & 1556 & 1529 & 1567 & 1498 \\
\hline
\end{tabular}
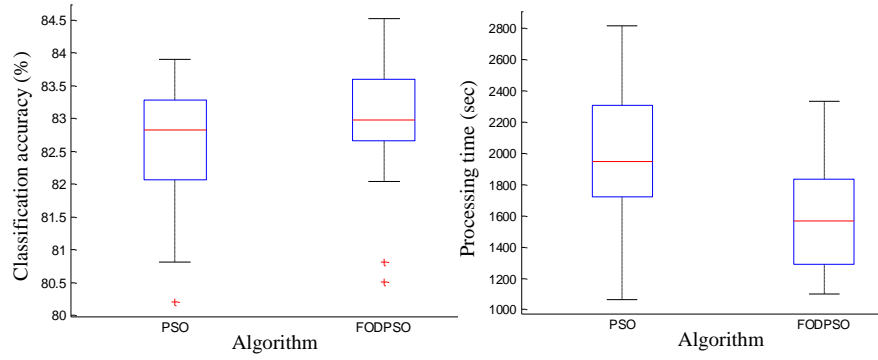

Fig. 11. Indian Pines in the second scenario: Final classification accuracy in percentage of the PSO- and FODPSO-based feature selection approaches.

The MANOVA analysis revealed that the type of algorithm led to a statistically significant different outcome on the multivariate composite $(F(1 ; 58)=18.8030 ; p$-value $<$ 0.0001). As the MANOVA detected significant statistical differences, we proceeded to the commonly-used ANOVA for each dependent variable. By carrying an individual test on each dependent variable, it was possible to observe that the OA does not present statistically significant differences $(F(1 ; 58)=3.3804 ; p-$ value $=0.0711)$. On the other hand, it is once again in the CPU processing time that both algorithms diverge the most, presenting statistically significant differences $(F(1 ; 58)=20.7238 ; p-$ value $<0.0001)$. As expected, the FODPSO-based approach (higher median value) produces slightly better solutions than the PSO and is considerably faster than the later.

To easily assess the differences between both algorithms, the outcome of each trial is graphically shown using box plot charts (Fig. 11). In the second scenario, one can easily observe the benefits of the fractional version of the algorithm, achieving a high level of OA in a short period of time.

Table. IV gives information regarding he number of selected features in Min, Median ${ }^{1}$, Median $^{2}$ and Max for different 
attributes, area (a) with 725 features, standard deviation (s) with 500 features, moment of inertia (i) with 450 features and diagonal of the box bounding the regions (d) with 500 features. As can be inferred from the table, the proposed method selects different number of features for different attributes in different runs. Therefore, it is difficult to conclude which attribute leads to better classification accuracies. However, it seems that the proposed methodology selects the highest number of features for the area attribute. The reason behind this might be that the area attribute is well related to the object hierarchy in the images and it generally can model the spatial information of images in a good way.

As can be seen from Table $\mathrm{V}$, the proposed feature selection technique has the best performance when the other feature selection and extraction techniques are not able to process the data due to the very high dimensionality and the limited number of training samples. All alternatives of the proposed method have almost the same performance in terms of OA and significantly improve on $A P$ and $R a w+A P$ in terms of classification accuracies. Both $A P$ and $R a w+A P$ dramatically suffer by the curse of dimensionality and the high redundancy of available features in the feature bank.

In addition, the proposed method can be considered as a good solution to overcome the shortcomings of attribute profiles. As can be seen, the proposed method can automatically find the most informative features from the feature bank including highly redundant features.

\section{CONCLUSION}

In this paper a novel feature selection approach is proposed, which is based on a new binary optimization technique named Binary FODPSO and SVM. The proposed approach was compared to commonly used feature selection and feature extraction approaches in experiments using standard AVIRIS hyperespectral data sets. Based on the experiments, the following points can be concluded:

- Binary FODPSO exploits many swarms in which each swarm individually performs just like an ordinary PSO algorithm with rules governing the collection of swarms that are designed to simulate natural selection. Moreover, the concept of fractional derivative is used to control the convergence rate of particles. The aforementioned reasons lead to a better performance than Binary PSO in terms of CPU processing time and $\mathrm{OA}$ for the cross-validation samples.

- In the novel feature selection approach, there is no need to set the number of output features and the proposed approach can automatically select the most informative features in terms of classification accuracies.

- Since the new approach is based on an evolutionary method, it is much faster than other well-known feature selection techniques which demand an exhaustive process to select the most informative bands. In this sense, the new approach can work appropriately in a situation which other feature selection techniques are not applicable.

- Since the new feature selection approach is based on a SVM classification which is capable of handling high dimensional data with a limited number of training samples, it can proceed to select the most informative features in an ill-posed situation when other feature selection/extraction techniques cannot proceed without a powerful technique for estimating the statistics for each class. As an example, when the original way is opted to estimate the statistics for each class, DBFE based on original statistics cannot proceed, since the number of pixels in the following classes needs to be at least one more than the total number of features being used, and LOOC statistics must be taken into account to handle this issue [1]. However, the new method can handle this problem effectively.

- The new approach can solve the main shortcomings of using AP for classification.

As a possible future work, we aim at finding the best SVM parameters (i.e., regularization and kernel parameters) by using the proposed Binary FODPSO in an automatic way instead of adjusting the parameters by using a cross-validation procedure after performing Binary FODPSO. In addition, in the second scenario, the proposed feature selection approach can be performed on each attribute profile separately, which generally leads to higher classification accuracy but in a higher CPU processing time. Therefore, another topic deserving future research is the development of parallel implementations of the presented approach in high-performance computing architectures, although the processing times reported in our experiments (measured in a standard desktop CPU) are quite small for the considered data sets.

\section{REFERENCES}

[1] D. A. Landgrebe, Signal Theory Methods in Multispectral Remote Sensing. Hoboken, NJ: Wiley, 2003.

[2] P. Ghamisi, J. A. Benediktsson, and J. R. Sveinsson, "Automatic spectral-spatial classification framework based on attribute profiles and supervised feature extraction," IEEE Trans. Rem. Sens. and Geos., In press.

[3] P. Ghamisi, J. A. Benediktsson, G. Cavallaro, and A. Plaza, "Automatic framework for spectral-spatial classification based on supervised feature extraction and morphological attribute profiles," IEEE Journal of Selected Topics in Applied Earth Observations and Remote Sensing, vol. PP, no. 99, pp. 1-1, 2014.

[4] G. Hughes, "On the mean accuracy of statistical pattern recognizers," IEEE Trans. Inf. Theory, vol. IT, no. 14, pp. $55-63,1968$.

[5] J. C. Russ, The Image Processing Handbook, 3rd ed. CRC Press LLC, 1999.

[6] D. J. Wiersma and D. A. Landgrebe, "Analytical design of multispectral sensors," IEEE Trans. on Geos. and Remote Sens., vol. GE, no. 18, pp. 180-189, 1980.

[7] P. Bajcsy and P. Groves, "Methodology for hyperspectral band selection," Photogramm. Eng. Remote Sens., vol. 70, no. 7, pp. 793-802, 2004.

[8] P. H. Swain, Fundamentals of pattern recognition in remote sensing. Remote Sensing-The Quantitative Approach, P. H. Swain and S. Davis, Eds. New York: McGraw-Hill, New York,, 1978. 
[9] K. Fukunaga, Introduction to Statistical Pattern Recognition. Academic Press, Inc., San Diego, California, 1990.

[10] X. Jia, B. Kuo, and M. M. Crawford, "Feature mining for hyperspectral image classification," Proceedings of the IEEE, vol. 101, no. 3, pp. 676-697, 2013.

[11] Y. Bazi and F. Melgani, "Toward an optimal svm classification system for hyperspectral remote sensing images," IEEE Trans. Geos. Remote Sens., vol. 44, no. 11, pp. 3374-3385, 2006.

[12] A. Daamouche, F. Melgani, N. Alajlan, and N. Conci, "Swarm optimization of structuring elements for vhr image classification," IEEE Geos. Remote Sens. Letters, vol. 10, no. 6, pp. 1334-1338, 2013.

[13] P. Ghamisi and J. A. Benediktsson, "Feature selection based on hybridization of genetic algorithm and particle swarm optimization," IEEE Geos. and Rem. Sens. Let., vol. 12, no. 2, pp. 309-313, 2015.

[14] A. Paoli, F. Melgani, and E. Pasolli, "Clustering of hyperspectral images based on multiobjective particle swarm optimization," IEEE Trans. Geos. Remote Sens., vol. 47, no. 12, pp. 4175-4188, 2009.

[15] F. vandenBergh and A. P. Engelbrecht, "A cooperative approach to particle swarm optimization," IEEE Trans. Evolutionary Computation, vol. 8, no. 3, pp. 225-239, 2004.

[16] K. Premalatha and A. Natarajan, "Hybrid pso and ga for global maximization," Int. J. Open Problems Compt. Math., vol. 2, no. 4, pp. 597-608, 2009.

[17] J. Kennedy and R. Eberhart, "A new optimizer using particle swarm theory," IEEE Sixth International Symposium on Micro Machine and Human Science, 1995.

[18] Y. D. Valle, G. K. Venayagamoorthy, S. Mohagheghi, J. C. Hernandez, and R. Harley, "Particle swarm optimization: Basic concepts, variants and applications in power systems," IEEE Trans. on Evolutionary Computation, vol. 2, no. 2, pp. 171-195, 2008.

[19] J. Tillett, T. M. Rao, F. Sahin, R. Rao, and S. Brockport, "Darwinian particle swarm optimization," Proceedings of the 2nd Indian International Conference on Artificial Intelligence, 2005.

[20] P. Ghamisi, M. S. Couceiro, J. A. Benediktsson, and N. M. F. Ferreira, "An efficient method for segmentation of images based on fractional calculus and natural selection," Expert Syst. Appl., vol. 39, no. 16, pp. $12407-$ $12417,2012$.

[21] P. Ghamisi, M. S. Couceiro, N. M. F. Ferreira, and L. Kumar, "Use of darwinian particle swarm optimization technique for the segmentation of remote sensing images," IGARSS, 2012.

[22] M. S. Couceiro, R. P. Rocha, N. M. F. Ferreira, and J. A. T. Machado, "Introducing the fractional order darwinian pso," Signal, Image and Video Processing, Springer, no. Fractional Signals and Systems Special Issue, 2012.

[23] P. Ghamisi, M. S. Couceiro, F. M. Martins, and J. A. Benediktsson, "Multilevel image segmentation approach for remote sensing images based on fractional-order darwinian particle swarm optimization," IEEE Trans. on of Remote Sens. and Geos., In press.

[24] M. S. Couceiro, F. M. Martins, R. P. Rocha, and N. M. Ferreira, "Mechanism and convergence analysis of a multi-robot swarm approach based on natural selection," Journal of Intelligent and Robotic Systems, pp. 1-29, 2014.

[25] M. A. Khanesar, M. Teshnehlab, and M. A. Shoorehdeli, "A novel binary particle swarm optimization," IEEE Mediterranean Conference on Control and Automation, pp. 1-6, 2007.

[26] V. N. Vapnic, Statistical Learning Theory. Hoboken, NJ: Wiley, 1998.

[27] B. Scholkopf and A. J. Smola, Learning with Kernels. MIT Press, 2002.

[28] M. Fauvel, J. Chanussot, and J. A. Benediktsson, "Kernel principal component analysis for the classication of hyperspectral remote-sensing data over urban areas," EURASIP Journal on Advances in Signal Processing, pp. $1-14,2009$.

[29] _ - "Evaluation of kernels for multiclass classification of hyperspectral remote sensing data," ICASSP, vol. 2, p. 813816, 2006.

[30] C.-W. Hsu and C.-J. Lin, "A comparison of methods for multiclass support vector machines," IEEE Trans. Neural Netw., vol. 13, no. 2, p. 415425, 2002.

[31] P. Ghamisi, M. Dalla Mura, and J. A. Benediktsson, "A survey on spectral-spatial classification techniques based on attribute profiles," IEEE Trans. Geos. Remote Sens., In press.

[32] M. Dalla Mura, J. A. Benediktsson, B. Waske, and L. Bruzzone, "Morphological attribute profiles for the analysis of very high resolution images," IEEE Trans. Geos. and Remote Sens., vol. 48, no. 10, pp. 3747-3762, 2010.

[33] — "Extended profiles with morphological attribute filters for the analysis of hyperspectral data," International Journal of Remote Sensing, vol. 31, no. 22, pp. 59755991, 2010.

[34] M. Fauvel, J. A. Benediktsson, J. Chanussot, and J. R. Sveinsson, "Spectral and spatial classification of hyperspectral data using SVMs and morphological profiles," IEEE Trans. Geos. and Remote Sens., vol. 46, no. 11, pp. 3804-3814, 2008.

[35] J. A. Peacock, "Two-dimensional goodness-of-fit testing in astronomy," pp. 615-627, 1983.

[36] A. C. Pedrosa and S. M. A. Gama, "Introducao computacional a probabilidade e estatistica," p. 348, 2004.

[37] J. Maroco, "Anlise estatstica com utilizao do spss," p. 199, 2010.

[38] J. Pallant, SPSS Survival Manual, 4th ed. Kindle Edition ed., Open University Press, 2011.

[39] C. Lee and D. A. Landgrebe, "Feature extraction based on decision boundaries," IEEE Transactions on Pattern Analysis and Machine Intelligence, vol. 15, no. 4, pp. 388-400, 1993. 


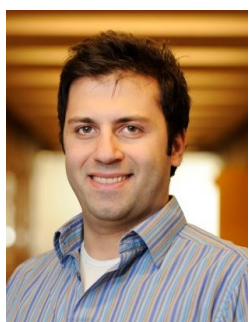

Pedram Ghamisi (S'12) graduated with a B.Sc degree in Civil (Survey) Engineering from the Tehran South Campus of Azad University. Then, he obtained the M.Sc. degree in Remote Sensing at K.N.Toosi University of Technology in 2012. He received the Best Researcher Award for M.Sc. students in $\mathrm{K}$. N. Toosi University of Technology in the academic year 2010-2011. At the 2013 IEEE International Geoscience and Remote Sensing Symposium (IGARSS), Melbourne, July 2013, Mr. Ghamisi was awarded the IEEE Mikio Takagi Prize, for winning the Student Paper Competition at the conference between approximately 70 people. He is currently a Ph.D. student in Electrical and Computer Engineering at the University of Iceland. His research interests are in remote sensing and image analysis with the current focus on spectral and spatial techniques for hyperspectral image classification and the integration of LiDAR and hyperspectral data for land cover assessment. He serves as a reviewer for a number of journals including IEEE TGRS, IEEE Trans. Image Processing, IEEE JSTARS, IEEE GRSL and Pattern Recognition Letter.

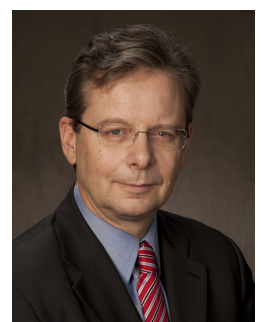

Jon Atli Benediktsson Jon Atli Benediktsson (S84M90SM99F04) received the Cand.Sci. degree in electrical engineering from the University of Iceland, Reykjavik, in 1984, and the M.S.E.E. and Ph.D. degrees from Purdue University, West Lafayette, IN, in 1987 and 1990, respectively. He is currently Pro-Rector of Science and Academic Affairs and Professor of Electrical and Computer Engineering at the University of Iceland. His research interests are in remote sensing, biomedical analysis of signals, pattern recognition, image processing, and signal processing, and he has published extensively in those fields. Prof. Benediktsson was the 2011-2012 President of the IEEE Geoscience and Remote Sensing Society (GRSS) and has been on the GRSS AdCom since 2000. He was Editor-in-Chief of the IEEE Transactions on Geoscience and Remote Sensing (TGRS) from 2003 to 2008 and has served as Associate Editor of TGRS since 1999, the IEEE Geoscience and Remote Sensing Letters since 2003 and IEEE Access since 2013. He is on the Editorial Board of the Proceedings of the IEEE, the International Editorial Board of the International Journal of Image and Data Fusion and was the Chairman of the Steering Committee of IEEE Journal of Selected Topics in Applied Earth Observations and Remote Sensing (J-STARS) 2007-2010. Prof. Benediktsson is a co-founder of the biomedical start up company Oxymap (www.oxymap.com). He is a Fellow of the IEEE and a Fellow of SPIE. Prof. Benediktsson is a member of the 2014 IEEE Fellow Committee. He received the Stevan J. Kristof Award from Purdue University in 1991 as outstanding graduate student in remote sensing. In 1997, Dr. Benediktsson was the recipient of the Icelandic Research Council's Outstanding Young Researcher Award, in 2000, he was granted the IEEE Third Millennium Medal, in 2004, he was a co-recipient of the University of Iceland's Technology Innovation Award, in 2006 he received the yearly research award from the Engineering Research Institute of the University of Iceland, and in 2007, he received the Outstanding Service Award from the IEEE Geoscience and Remote Sensing Society. He was co-recipient of the 2012 IEEE Transactions on Geoscience and Remote Sensing Paper Award and in 2013 he was co-recipient of the IEEE GRSS Highest Impact Paper Award. In 2013 he received the IEEE/VFI Electrical Engineer of the Year Award. In 2014, he was a co-recipient of the 2012-2103 Best Paper Award from the International Journal of Image and Data Fusion. He is a member of the Association of Chartered Engineers in Iceland (VFI), Societas Scinetiarum Islandica and Tau Beta Pi.

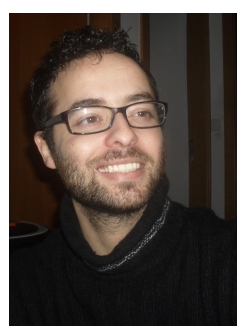

Micael Couceiro obtained the BSc, Teaching Licensure and Master degrees on Electrical Engineering (Automation and Communications), at the Engineering Institute of Coimbra, Polytechnic Institute of Coimbra (ISEC-IPC). He obtained the PhD degree on Electrical and Computer Engineering (Automation and Robotics) at the Faculty of Sciences and Technology, University of Coimbra (FCTUC) in April 2014. Over the past 6 years, he has been conducting scientific research on several areas besides robotics, namely computer vision, sports engineering, economics, sociology, digital media, and others, all at the Institute of Systems and Robotics (ISR-UC), and at RoboCorp research group from IPC. This resulted on more than 20 scientific articles in international impact factor journals and more than 40 scientific articles at international conferences. Besides research, he has been invited for lecturing, tutoring and organization of events (e.g., professional courses, national and international conferences, among others), both in the public and private domains. He is currently the CEO of Ingeniarius, Lda. 